\title{
A Rule-Based Approach to XML Processing and Web Reasoning *
}

\author{
Jorge Coelho $^{1}$, Besik Dundua ${ }^{2}$, Mário Florido $^{2}$, and Temur Kutsia ${ }^{3}$ \\ 1 ISEP \& LIACC, Porto, Portugal \\ jcoelho@liacc.up.pt \\ 2 DCC-FC \& LIACC, University of Porto, Portugal \\ $\{$ bdundua, amf\}@dcc.fc.up.pt \\ 3 RISC, Johannes Kepler University, Linz, Austria \\ kutsia@risc.uni-linz.ac.at
}

\begin{abstract}
We illustrate the potential of conditional hedge transformations in Web-related applications on the example of $\mathrm{P} \rho \mathrm{Log}$ : an extension of logic programming with advanced rule-based programming features for hedge transformations, strategies, and regular constraints.
\end{abstract}

\section{Introduction}

The rule-based approach has been used extensively in many fields, such as expert systems, theorem proving, tree automata, software building and configuration, banking systems, just to name a few. In recent years, the rule-based approach has been experiencing growing popularity in Web applications. One could mention document processing and Web reasoning as prominent examples. The REWERSE project [23] provides an extensive reference material on those topics.

The goal of this paper is to illustrate the potential of strategy-based conditional hedge transformations in Web-related applications. To achieve this goal, first, we present a practical tool: an extension of logic programming with advanced rule-based programming features for hedge transformations, strategies, and regular constraints. Second, we show how it can be used it XML querying, validation, and some basic Web reasoning.

The tool we describe here is $\mathrm{P} \rho \log [13]$ (pronounced Pē-rō-log). It is a Prolog implementation of the $\rho \log$ calculus [19], which extends the host language with strategic conditional transformation rules. These rules (basic strategies) define transformation steps on hedges. (A hedge is a sequence of unranked terms.) Strategy combinators help to combine strategies into more complex ones in a declaratively clear way. Transformations are nondeterministic and may yield several results, which fits very well into the logic programming paradigm. Strategic

\footnotetext{
* Partially funded by LIACC through Programa de Financiamento Plurianual of the Fundação para a Ciência e Tecnologia (FCT), by the FCT fellowship (ref. SFRH/BD/62058/2009), and by the EC FP6 for Integrated Infrastructures Initiatives under the project SCIEnce (contract No. 026133).
} 
rewriting separates term traversal control from transformation rules. The separation of strategies and rules makes rules reusable in different transformations.

$\mathrm{P} \rho \mathrm{Log}$ programs consist of clauses. The clauses either define user-constructed strategies by (conditional) transformation rules or are ordinary Prolog clauses. Prolog code can be used freely within $\mathrm{P} \rho \log$ programs, which is especially convenient when arithmetic calculations or input-output features are needed.

$\mathrm{P} \rho \mathrm{Log}$ uses four different kinds of variables, which permits to traverse hedges in single/arbitrary width (with individual and sequence variables) and terms in single/arbitrary depth (with functional and context variables). It provides a possibility to extract an arbitrary subhedge from a hedge, or to extract subterms at arbitrary depth. In addition, $\mathrm{P} \rho \log$ permits regular constraints to restrict possible values of sequence and context variables by regular hedge expressions and regular tree (context) expressions, respectively. These constraints are very useful, for instance, in validation of an XML document with respect to a given DTD.

$\mathrm{P} \rho \mathrm{Log}$ has not been implemented specifically for Web-related applications. Its main purpose is to bring strategy-based conditional hedge transformations in the logic programming framework for general programming. The role of $\mathrm{P} \rho \mathrm{Log}$ in this paper is to provide a practical platform to illustrate suitability of the calculus behind it in XML querying, validation, and Web reasoning.

In the context of XML processing, the approach $\mathrm{P} \rho \log$ is based on can be classified as positional or pattern-based, where programmer specifies patterns including variables. Examples of such languages are Xcerpt [7], UnQL [8], XDuce [15], and CDuce [4]. Usually, in this approach, variables in patterns specify the nodes to be selected. With $\mathrm{P} \rho \mathrm{Log}$, we can select not only nodes but also sequences of nodes, node labels, and the context around a node that is at arbitrary depth. Moreover, it can naturally express incomplete query patterns.

Approaches to XML, based on the logic programming paradigm, have been quite popular. Besides the already mentioned Xcerpt, the languages like Elog [3], XPathLog [21], and XCentric [11,12] belong to this category. The latter one, like $\mathrm{P} \rho \mathrm{Log}$, represents XML data as an unranked Prolog term and uses sequence matching with regular types for querying. In fact, for our experiments we used XCentric's XML-to-unranked-term translator.

From the general programming point of view, we should mention a number of calculi and languages for rule- and strategy-based programming related to our work, such as rewriting logic [20], $\rho$-calculus [9], ASF-SDF [25], CHR [14], ELAN [6], Maude [10], the OBJ family of languages [22], Stratego [26], and TOM [2]. $\rho \log$, the calculus behind $\mathrm{P} \rho \log$, has been influenced by the $\rho$-calculus. However, there are specific features in $\rho$ Log that makes it significantly different from the $\rho$-calculus: logic programming semantics, top-position matching, hedge transformations, different kinds of variables, and regular constraints.

\section{$2 \quad \mathrm{P} \rho \log$}

In this section we give a brief overview of basic features of $\mathrm{P} \rho \log$, explaining them mostly on examples instead of giving formal definitions. 
Terms and hedges (sequences of terms) in $\mathrm{P} \rho \mathrm{Log}$ are built over unranked function symbols and four kinds of variables: individual, sequence, function, and context variables. These sets are disjoint. In this paper we follow the $\mathrm{P} \rho \mathrm{Log}$ notation for this language, writing its constructs in typewriter font. $\mathrm{P} \rho \mathrm{Log}$ uses the following conventions for the variables names: Individual variables start with $i_{-}$(like, e.g., i_Var for a named variable or $i_{-}$for the anonymous variable), sequence variables start with $\mathrm{s}_{-}$, function variables start with $\mathrm{f}_{-}$, and context variables start with $c_{-}$. The function symbols, except the special constant hole, have flexible arity. To denote function symbols, $\mathrm{P} \rho \log$ basically follows the Prolog conventions for naming functors, operators, and numbers. Terms $t$ and hedges $\mathrm{h}$ are constructed by the grammars:

$t::=i_{-} X|f(h)| f_{-} X(h)\left|c_{-} X(t) \quad h::=t\right| s_{-} X \mid$ eps $\mid$ (h_1, h_2)

where eps stands for the empty hedge and is omitted whenever it appears as a subhedge of another hedge. a (eps) and $f_{-} X(e p s)$ are often abbreviated as a and $f_{-} X$. A Context is a term with a single occurrence of hole. A context $\mathrm{C}$ can be applied to a term $t$, written $\mathrm{C}[\mathrm{t}]$, replacing the hole in $\mathrm{C}$ by $\mathrm{t}$.

A substitution is a mapping from individual variables to hole-free terms, from sequence variables to hole-free hedges, from function variables to function variables/symbols, and from context variables to contexts, such that all but finitely many individual, sequence, and function variables are mapped to themselves, and all but finitely many context variables are mapped to themselves applied to the hole. This mapping is extended to terms/hedges in the standard way.

Matching problems are pairs of hedges, one of which is ground (i.e., does not contain variables). Such matching problems may have zero, one, or more (finitely many) solutions, called matching substitutions or matchers. For instance, the hedge $\left(s_{-} 1, f\left(i_{-} X\right), s_{-} 2\right)$ matches $(f(a), f(b), c)$ in two different ways: one by the matcher $\left\{s_{-} 1 \mapsto e p s, i_{-} X_{\mapsto} \rightarrow a, s_{-} 2 \mapsto(f(b), c)\right\}$, the other one by the matcher $\left\{\mathrm{s}_{-} 1 \mapsto f(a), i_{-} X \mapsto b, s_{-} 2 \mapsto c\right\}$. Similarly, the term $c_{-} X\left(f_{-} Y(a)\right)$ matches the term $f(a, g(a))$ with the matchers $\left\{c_{-} X \mapsto f(h o l e, g(a)), f_{-} Y \mapsto f\right\}$ and $\left\{c_{-} X \mapsto f(a, g(h o l e)), f_{-} Y \mapsto g\right\}$. An algorithm to solve matching problems in the described language has been introduced in [17].

Instantiations of sequence and context variables can be restricted by regular hedge and regular context languages, respectively, specified by the corresponding regular expressions. We do not go into the details of the regular constraints here, just mention that they can be added to matching problems to restrict the set of computed matchers, e.g., matching $c_{-} X\left(f_{-} Y(a)\right)$ to $f(a, g(a))$ under the constraint $c_{-} X$ in $f\left(a, g(h o l e)^{\star}\right)$ gives one matcher $\left\{c_{-} \mathrm{X} \mapsto f(a, g(h \circ l e)), f_{-} Y \mapsto g\right\}$ instead of two for the unconstrained case.

A $\rho \log$ atom ( $\rho$-atom) is a quadruple consisting of a hole-free term st (a strategy), two hole-free hedges $\mathrm{h} 1$ and $\mathrm{h} 2$, and a set of regular constraints $\mathrm{R}$ where each variable is constrained only once, written as st : : h1 ==> h2 where R. Intuitively, it means that the strategy st transforms $\mathrm{h} 1$ to $\mathrm{h} 2$ when the variables satisfy the constraint $R$. When $R$ is empty, we omit it and write st $::$ h1 $==>$ h2. The negated atom is written as st : : h1 $=\backslash \Rightarrow$ h2 where R. A $\rho$ Log literal ( $\rho$ literal) is a $\rho$-atom or its negation. A P $\rho \log$ clause is either a Prolog clause, or 


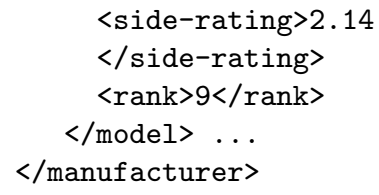

We assume that sequences of these elements are wrapped respectively by <list-manuf> and <list-vehicle> tags. To save space, in the queries below we use metavariable $M$ to refer to the document with the root tag <list-manuf> and $V$ to the document with the root tag <list-vehicle>.

Selection and Extraction: We want to select and extract <manufacturer> elements where some <model> has <rank> less or equal to 10.

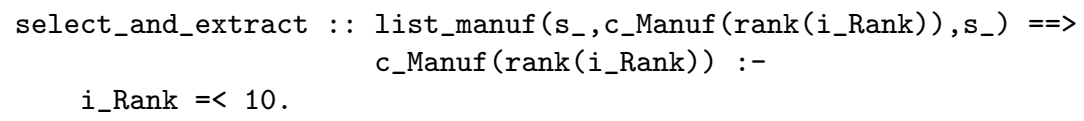

Given the goal select_and_extract : : $M==>$ i_M, this code generates all solutions, one after the other, via backtracking. The alternatives are generated according how list_manuf ( $\mathbf{s}_{-}, \mathrm{c}_{-} \operatorname{Manuf}\left(\operatorname{rank}\left(i_{-}\right.\right.$Rank $\left.\left.)\right), \mathrm{s}_{-}\right)$matches $M$.

Reduction: From the <manufacturer> elements, we want to drop the <model> subelements whose $\langle$ rank $\rangle$ is greater than 10 . Besides that, we also want to elide the <front_rating > and <side_rating > elements from the remaining models. It can be done in various ways in $\mathrm{P} \rho \mathrm{Log}$. One of such implementations is given below. reduction is defined as the normal form of transforming each manufacturer element inside list_manuf. A single manufacturer element is transformed by reduction_step depending whether it contains a model with the rank $\leq 10$ :

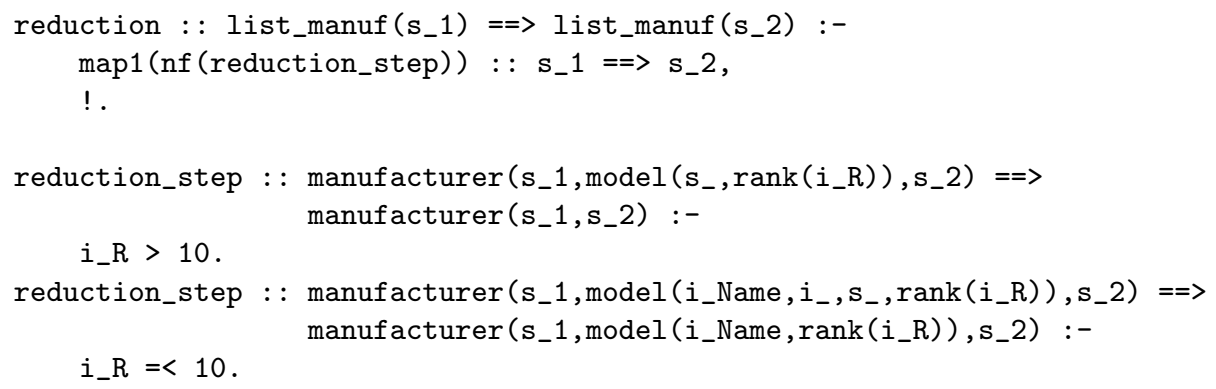

Here $\mathrm{nf}$ is the built-in strategy for a normal form computation. Another builtin strategy, map1, maps its argument strategy to each single term of the input hedge. The query reduction : : $M==>$ i_List produces the list of reduced manufacturer elements.

Incomplete Queries. Often, the structure of a document to be queried is unknown to a query author, or she is interested not in the entire document but only in its relevant parts. A pattern-based Web querying language should 
be able to express such incomplete queries. Schaffert in [24] classifies incomplete queries (four kinds of incompleteness: in breadth, in depth, with respect to order and with respect to optional elements) and explains how they are dealt with in Xcerpt. Here we show how they can be expressed in $\mathrm{P} \rho \mathrm{Log}$. As we will see, it can be done pretty naturally, without introducing additional constructs for them.

Incompleteness in breadth. In languages that have wildcards only for single terms, expressing incompleteness in breadth requires a special construct that allows to omit those wildcards for neighboring nodes in the data tree. In $\mathrm{P} \rho \mathrm{Log}$, we do not need any extra construct because of sequence variables. Anonymous sequence variables can be used as wildcards for arbitrary sequence of nodes. Furthermore, if needed, we can use named sequence variables to extract arbitrary sequence of nodes without knowing the exact structure. These are very convenient features, as one can see from the examples in the previous section.

Incompleteness in depth. It allows to select data items that are located at arbitrary, unknown depth and skip all structure in between. For this, in $\mathrm{P} \rho \mathrm{Log}$ we just have to place the corresponding query subterm under an anonymous context variable. Moreover, if needed, we can extract the entire context above the query subterm without knowing the structure of the context, by putting there a named context variable. This has been done in the select_and_extract clause in the previous section with the c_Manuf variable. In fact, that clause also demonstrates how $\mathrm{P} \rho \log$ can combine incompleteness in breadth and depth in a single rule.

Incompleteness with respect to order. It allows to specify neighboring nodes in a different order than the one in that they occur in the data tree. Since P $\rho$ Log does not permit matching in orderless theories ${ }^{4}$ we need a bit of more coding here. Assume that we do not know in which order the front_rating and side_rating elements occur in the model in Example 1 and write the clause that extract them:

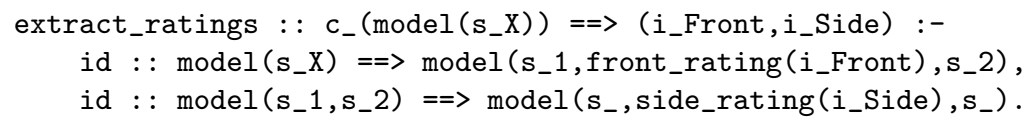

In the first subgoal of the body of this rule, the id strategy forces the term model (s_1, front_rating(i_Front), s_2) to match model (s_X), extracting the value for front rating i_Front. Next, to find the side rating, we force matching model ( $\mathbf{s}_{-}$, side_rating (i_Side), $\mathbf{s}_{-}$) to model (s_1, $\mathbf{s}_{-} 2$ ) that is obtained from model(s_X) by deleting front_rating(i_Front). This deletion comes for free from the previous match and we can take an advantage of it, since there is no need to keep front_rating in the structure where side_rating is looked for.

Incompleteness with respect to optional elements. Since seq. variables can be instantiated with the empty hedge, such queries are trivially expressed in $\mathrm{P} \rho \mathrm{Log}$.

\footnotetext{
4 The orderless property is a generalization of commutativity for unranked function symbols. For orderless matching over unranked terms, see [16].
} 


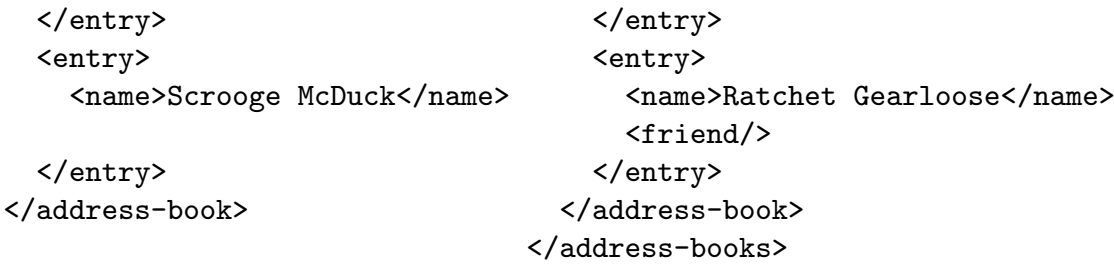

The collection contains two address books, the first owned by Donald Duck and the second by Daisy Duck. Donalds address book has two entries, one for Scrooge, the other for Daisy, and only Daisy is marked as friend. Daisys address book again has two entries, both marked as friend.

The clique-of-friends of Donald is the set of all persons that are either direct friends of Donald (i.e. in the example above only Daisy) or friends of a friend (i.e. Gladstone and Ratchet), or friends of friends of friends (none in the example above), and so on. To retrieve these friends, we have to define the relation "being a friend of" and its transitive closure.

Transitive closure of a relation can be easily defined in $\mathrm{P} \rho \mathrm{Log}$. It can be even written in a generic way, parameterized by the strategy that defines the relation:

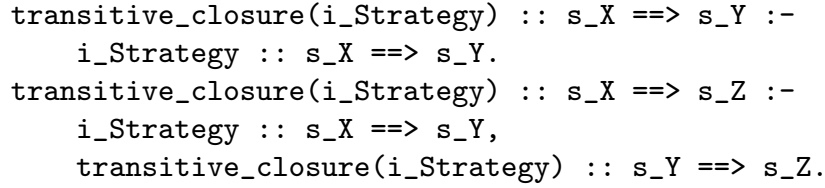

The relation of "being a friend of" with respect to the address books document is defined as follows:

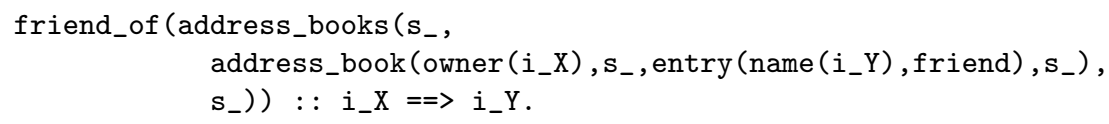

The query transitive_closure(friend_of $(T)$ ) : : Donald_Duck ==> i_Y, where $T$ is the $\mathrm{P} \rho \mathrm{Log}$ term corresponding to the address book XML document above, will return one after the other the friend and the friends of the friend of Donald_Duck: Daisy_Duck, Gladstone_Duck, and Ratchet_Gearloose.

\section{References}

1. K. R. Apt and R. Bol. Logic programming and negation: A survey. J. Logic Programming, 19:9-71, 1994.

2. E. Balland, P. Brauner, R. Kopetz, P.-E. Moreau, and A. Reilles. Tom: Piggybacking rewriting on Java. In Proc. RTA'0\%, volume 4533 of LNCS, pages 36-47. Springer, 2007.

3. R. Baumgartner, S. Flesca, and G. Gottlob. The Elog web extraction language. In Proc. LPAR'01, volume 2251 of $L N C S$, pages 548-560. Springer, 2001.

4. V. Benzaken, G. Castagna, and A. Frisch. CDuce: an XML-centric general-purpose language. In Proc. ICFP'03, pages 51-63. ACM, 2003. 
5. A. Bonifati and S. Ceri. Comparative analysis of five XML query languages. $A C M$ SIGMOD Record, 29(1):68-79, 2000.

6. P. Borovanský, C. Kirchner, H. Kirchner, P.-E. Moreau, and M. Vittek. Elan: A logical framework based on computational systems. ENTCS, 4, 1996.

7. F. Bry and S. Schaffert. Towards a declarative query and transformation language for XML and semistructured data: Simulation unification. In Proc. ICLP'02, number 2401 in LNCS. Springer, 2002.

8. P. Buneman, M. Fernandez, and D. Suciu. UnQL: a query language and algebra for semistructured data based on structural recursion. The VLDB Journal, 9(1):76$110,2000$.

9. H. Cirstea and C. Kirchner. The rewriting calculus - Parts I and II. Logic Journal of the IGPL, 9(3), 2001.

10. M. Clavel, F. Durán, S. Eker, P. Lincoln, N. Martí-Oliet, J. Meseguer, and J. F. Quesada. Maude: specification and programming in rewriting logic. Theoretical Computer Science, 285(2):187-243, 2002.

11. J. Coelho and M. Florido. CLP(Flex): Constraint logic programming applied to XML processing. In CoopIS, DOA, and ODBASE 2004, volume 3291 of LNCS, pages 1098-1112. Springer, 2004.

12. J. Coelho and M. Florido. XCentric: A logic programming language for XML. Technical Report DCC-2005-X, DCC-FC and LiACC, University of Porto, 2005.

13. B. Dundua and T. Kutsia. P $\rho$ Log. Version 0.7. Available from: http://www.risc.uni-linz.ac.at/people/tkutsia/software.html.

14. T. Frühwirth. Theory and practice of Constraint Handling Rules. J. Logic Programming, 37(1-3):95-138, 1998.

15. H. Hosoya and B. C. Pierce. XDuce: A statically typed XML processing language. ACM Trans. Internet Techn., 3(2):117-148, 2003.

16. T. Kutsia. Solving and Proving in Equational Theories with Sequence Variables and Flexible Arity Symbols. PhD thesis, Johannes Kepler University, Linz, 2002.

17. T. Kutsia and M. Marin. Matching with regular constraints. In $L P A R$ '05, volume 3835 of $L N A I$, pages 215-229. Springer, 2005.

18. D. Maier. Database desiderata for and XML query language. Available from: http://www.w3.org/TandS/QL/QL98/pp/maier.html, 1998.

19. M. Marin and T. Kutsia. Foundations of the rule-based system RhoLog. Journal of Applied Non-Classical Logics, 16(1-2):151-168, 2006.

20. N. Martí-Oliet and J. Meseguer. Rewriting logic: Roadmap and bibliography. Theoretical Computer Science, 285(2):121-154, 2002.

21. W. May. XPath-Logic and XPathLog: a logic-programming-style XML data manipulation language. TPLP, 4(3):239-287, 2004.

22. The OBJ Family. http://cseweb.ucsd.edu/ goguen/sys/obj.html.

23. REWERSE. Reasoning on the web. http://rewerse.net/.

24. S. Schaffert. Xcerpt: a rule-based query and transformation language for the Web. PhD thesis, University of Munich, 2004.

25. M. G. J. van den Brand, A. van Deursen, J. Heering, H. A. de Jong, M. de Jonge, T. Kuipers, P. Klint, L. Moonen, P. A. Olivier, J. Scheerder, J. J. Vinju, E. Visser, and J. Visser. The ASF + SDF meta-environment: A component-based language development environment. In $C C^{\prime} 01$, volume 2027 of $L N C S$, pages 365-370. Springer, 2001.

26. E. Visser. Stratego: A language for program transformation based on rewriting strategies. In $R T A$ '01, volume 256 of $L N C S$, pages 357-362. Springer, 2001. 